\title{
Rational Selection of Rail Transit Emergency Site Using Complex Network Topology and Genetic Algorithm
}

\author{
Jing Zhou 1 and Yunhong Shao \\ Zhejiang Institute of Communications, Hangzhou 311112, China \\ Correspondence should be addressed to Jing Zhou; zhoujingtrans1988@163.com
}

Received 9 November 2021; Revised 5 January 2022; Accepted 15 January 2022; Published 24 February 2022

Academic Editor: Rahman Ali

Copyright (C) 2022 Jing Zhou and Yunhong Shao. This is an open access article distributed under the Creative Commons Attribution License, which permits unrestricted use, distribution, and reproduction in any medium, provided the original work is properly cited.

\begin{abstract}
Rail transit network is a convenient and reliable transportation mode in urban areas. With every surge in transportation demand, the network undergoes operational changes like restructuring and making of multiline tracks. Urban rail transit network is a complex network and needs proper emergency services and controlling mechanisms. Unexpected mishaps in rail transit will cause more hazard and panic than the other transportation systems. Therefore, a reliable emergency service station is required to ensure the safety and security of passengers. In the proposed method, the basic statistical properties of complex network topology are considered to construct a $P$-center site selection model for urban rail transit emergency service stations. The $P$-center site selection model is solved by genetic algorithm. Validity and reasonableness of the model are demonstrated by implementing it in the Hangzhou emergency service stations for rail transportation. The results show that the $P$-center site selection model based on statistical properties of complex network topologies can better solve the urban rail transit emergency service station site selection problems. The model minimizes the number of emergency service stations while satisfying the optimal objective function and reduces the construction cost of emergency service stations. The approach has a significant effect on improving system reliability and reducing the risk of emergencies.
\end{abstract}

\section{Introduction}

At present, urban rail transit is in a period of rapid development, strategic development, and management transition. The scale of line network size of major cities continues to expand. The benefits of network scale are becoming increasingly apparent as the challenges of security management increase. At any network station, different sorts of emergency events might occur unexceptedly. If not schemed and forecast properly, the difficulties in managing destructiveness, chain hazard, and rescue issues will enormously increase. Once an unexpected emergency event occurs at any station in the network, it will cause a systemic extended impact. However, the exponential difficulties of urban rail transit emergency events can be handled through an intelligent response system. Therefore, setting up of emergency service stations scientifically and rationally has become a hot topic for experts and scholars. To minimize economic losses and casualties in such accidents, the emergency station is supposed to provide initiate rescue processes promptly and fittingly.

The issue of rescue site selection is an important topic for emergency resourcing research in safety management [1]. As early as 1950, Valinsky [2] proposed a model for fire emergency rescue service site selection. The study shows that most emergency facility site selections are nondeterministic polynomial (NP) problem. In 1964, Hakimi $[3,4]$ was the first to propose a special discrete site model, the $P$-center model, to solve the emergency rescue service site selection problem. The model is an effective method to solve the NP problem, and is therefore, a milestone in the history of emergency rescue service site selection development. In 1979, Toregas et al. [5] proposed a model for emergency rescue service site selection based on set coverage model. Both the set coverage model and the $P$-center model are global coverage models that are more optimal than local 
coverage. The models are widely used in problems, such as the site of logistics distribution centers, highway emergency rescue centers, and medical emergency center. As the research continues, more and more scholars are optimizing the model based on the previous studies and outcomes. The main aspects of optimization include the construction of multiobjective function models (e.g., minimization of rescue time and minimization of rescue cost), decision models that consider uncertainty factors (e.g., sharing uncertainty factors and demand uncertainty factors), and dynamic site selection model [6]. For example, Duan and Xiaowan [7] developed a maximum coverage model with station importance as the key factor and with the optimal emergency of response time and emergency rescue stations number as the decision objective. The congestion comparison algorithm and tournament selection algorithm are pertinent to mention. The models converge faster and lead to better site selection results. Wang and Zhou [8] divided the railroad rescue base into two levels: rapid rescue base and regional rescue base. The method presents an absolute center site selection model that takes into account the uncertainty of rescue demand to achieve rapid site selection and meet the differentiated service of rescue demand. Fan et al. [9] considered the undecided demands and construction cost and node failure to cope with inland port site selection problem. The authors designed a robust stochastic site selection model. In the model, the stochastic programming theory is applied to solve the problem of demand uncertainty. The minimum and maximum regret value theory is applied to solve the problem of construction cost uncertainty. A hybrid algorithm combining particle swarm algorithm and simulated annealing algorithm is designed to solve the problem. Validity of the model is verified by example analysis. In transportation field, unlike logistics, railroads, highways, ports, and other modes of transportation, the network operation process of urban rail transportation is scarcely focused. The emergency rescue service site selection problem in urban rail system has not yet been reviewed systematically. Peeters and Thomas [10] show that the network structure has a significant impact on facility siting. Chai et al. [11] proposed a valuable model to efficiently define candidate lines for safe pass through of rails. In the model of Fielbaum et al. [12] four transit structures are suggested for safe and speedy transportation. The method by Liu and Yuan [13] exploited fuzzy network analysis to deal with emergency in rail transit system. Similarly, for risk and emergency management, Cadarso et al. [14] proposed a model based on risk-averse strategies.

Various models in the literature have been proposed for site selection of urban rail transit emergency service stations. The models mainly exploit local coverage problem and focus only on rescue response time and distance. However, the urban rail transit emergency rescue should be treated as a global coverage problem. This research work utilizes genetic algorithm and global convergence to satisfy the optimal objective function and reduces the construction cost of emergency. By considering the topological structure characteristics of the network, a $P$-center siting model is constructed for urban rail transit emergency rescue stations.
Considering the basic characteristics of complex network topology and utilizing genetic algorithm, the model attempts to solve the optimization problem. Effectiveness and rationality of the model is demonstrated by taking siting emergency rescue stations of Hangzhou rail transit as an example. The outcomes suggest that the model is quite applicable in selecting rescue stations for urban rail transit system.

\section{Basic Properties of Complex Networks}

There is no unified definition of complex networks; however, the Qian Xuesen's [15] description of complex networks is pertinent to start with. According to Qian Xuesen, networks with some or all of the properties of self-organization, selfsimilarity, attractors, small worlds, and scale-free are referred to as complex networks [15]. Complex networks are generally characterized by complexity of structure, connection structure, diversity of nodes, variation of edges, and dynamic evolution of structure. Urban rail transit system is usually abstracted as a complex network, with each station acting as a node and the interaction between stations described as an edge in the network. The basic characteristics of the network and the practical applications of urban rail transit systems are further analyzed. In a complex network topology, a series of metrics such as degree and its distribution, betweenness centrality, path length, clustering coefficient, and network efficiency are the specific reflections of the basic characteristics [16]. In the following section, we describe the metric values, mediators, and path lengths involved in the text.

2.1. Degree. The degree $k_{i}$ of node $i$ is a local statistical characteristic parameter in complex networks, it is the number of nodes connected to node $i$. In a directed network, the degree can be divided into two types: out-degree and indegree. The out-degree indicates the number of edges that point from the node itself to other nodes in the network, and the in-degree indicates the number of edges that are pointed to the node itself by other nodes in the network. By definition, there is a clear positive correlation between the degree of a node and its importance in the network. In the urban rail transit network system, the degree values of interchange stations, intermediate stations, and the first or last stations are 3 and above, 2, and 1, respectively. The complexity of networked operations varies, as does the maximum degree value of the site.

$$
\begin{gathered}
k_{i}=\sum_{j \in N} a_{i j}, \\
\langle k\rangle=\frac{1}{N} \sum_{i=1}^{N} k_{i},
\end{gathered}
$$

where $k_{i}$ is the degree value of node $i,\langle k\rangle$ is the average degree value of the network, $a_{i j}$ is the number of nodes (sites) connected to node $i$, and $N$ is the total number of network nodes. 
2.2. Betweenness Centrality. The betweenness centrality is a measure of a node's centrality in a network equal to the number of shortest paths from all vertices to all others that pass through that node. Betweenness centrality is a more useful measure of the load placed on the given node in the network as well as the node's importance to the network than just its connectivity. The latter is only a local effect while the former is more global to the network. In the urban rail transit network system, the larger the betweenness centrality of stations, the greater its role in the process of passenger flow transmission and the greater the carrying capacity of passenger flow.

$$
\begin{aligned}
& \mathrm{Bn}=\sum_{i \neq j \in N} \frac{\sigma_{i j}(n)}{\sigma_{i j}}, \\
& \mathrm{Be}=\sum_{i \neq j \in N} \frac{\sigma_{i j}(e)}{\sigma_{i j}},
\end{aligned}
$$

where $\mathrm{Bn}$ is the betweenness centrality of node $n, \mathrm{Be}$ is the betweenness centrality of edge $e, \sigma_{i j}$ is the number of shortest paths between nodes $i$ and $j, \sigma_{i j}(n)$ is the number of shortest paths between nodes $i$ and $j$ that pass through node $n$, and $\sigma_{i j}(e)$ is the number of shortest paths between node $i$ with node $j$ that pass through edge $e$.

2.3. Shortest Path. The path from node $i$ to node $j$ that passes through the least number of edges is called the shortest path, and the number of edges in that path is called the shortest path length. From a global view of the network, the average path length of the road network can be used to measure the degree of dispersion between individual nodes. The equation for calculating the average path length of the road network is shown in equation (3).

$$
L=\frac{1}{n(n-1)} \sum_{i, j \in V(i \neq j)} d_{i j} .
$$

In the above equation, $d_{i j}$ is the shortest path length from node $i$ to node $j$.

\section{Site Selection Model and Solution Based on Complex Network Characteristics}

The key characteristics of the complex network of rail transit system are considered in the construction of the model. Details are as follows.

3.1. Model Matching. The common siting models mainly set the coverage model, maximum coverage model, $P$-median model, and $P$-center model, and so on. Different site selection models consider different central issues. The core ideas and applicable conditions of the commonly used model are comparatively analyzed. Results of the analysis are shown in Table 1.

By comparing the above common siting models, it is apparent that each model takes into account the coverage, emergency response time, rescue distance and cost, but each model has a different focus. Maximum coverage model is a local rescue coverage problem that focuses on maximizing the use of established resources. Both the collective coverage model and the $P$-center model can achieve full rescue coverage. A premise of the former is the assumption that the capacity of the service facility is sufficient. This means that no matter how large the emergency service demand is, it can be met by the facility point covering it. The latter focuses on global worst-case optimization, which is a more rigorous "economic equilibrium" model $[17,18]$. The $P$-median model is focused on the global rescue average optimal, given the established resources. Urban rail transit emergency rescue should be treated as a global coverage problem. The timeliness, effectiveness, and minimization of system loss or impact of rescue are the key points to be considered for the urban rail transit emergency models. Therefore, the $P$-center model can be used to solve the problem of siting urban rail transit emergency rescue sites.

3.2. Model Building. Objective of this study is to propose an intelligent model for rail transit emergency site selection. Details about the model are as follows.

3.2.1. Emergency Service Demand Sites. Suppose there are $m$ stations in the urban rail network, each station may be an emergency service demand site, namely sudden accident and occurrence point. The emergency rescue sites can be can be expressed as a set $U=\left\{u_{1}, u_{2}, u_{3}, \ldots, u_{m}\right\}$.

3.2.2. Emergency Rescue Alternative Sites. Emergency rescue alternative sites can be expressed as a set $V=\left\{v_{1}, v_{2}, v_{3}, \ldots, v_{n}\right\} \subset U$. Sites that meet the following conditions can be included in the alternative sites. The conditions are as follows. (1) Interchange stations with a degree value of not less than 2 and high betweenness centrality. (2) The nodes taken should be relatively important nodes of the network. (3) Parking lot (vehicle section) or the nearest station to the parking lot (vehicle section), they have large land space, which can reduce the land occupation and construction cost. (4) Interchange hubs and integrated transportation feeder sites, such as train stations, bus stations, large shopping malls, and other points where they are located, tend to have a large passenger flow and a large loss after the occurrence of emergencies.

3.2.3. Emergency Rescue Mandatory Sites. Emergency rescue mandatory sites can be expressed as a set $T=\left\{v_{n 1}, v_{n 2}, v_{n 3}, \ldots, v_{n t}\right\} \subset V \subset U$, the mandatory sites are the important nodes in the network, which are selected based on the characteristics of the complex network such as site degree and betweenness centrality.

Suppose, emergency rescue demand sites (sudden accident occurrence point) and emergency rescue service sites are the urban rail network nodes. In such cases, emergency rescue response is conducted along the shortest distance of the urban rail line, without regard to other modes of transportation. If the existing emergency rescue sites are not 
TABLE 1: Comparison analysis of site selection model comparison analysis.

\begin{tabular}{|c|c|c|}
\hline Site selection model & Core ideas & Applicable conditions \\
\hline $\begin{array}{lc} & \text { Response } \\
\text { Set coverage } & \text { mechanism model } \\
\text { model } & \text { Anchoring the } \\
& \text { mandatory model }\end{array}$ & $\begin{array}{c}\text { Emergency rescue service time or distance in meeting } \\
\text { the established requirements, with a minimum } \\
\text { number of service stations to cover the entire } \\
\text { emergency demands. }\end{array}$ & $\begin{array}{l}\text { Requirements such as emergency response } \\
\text { time or distance are more stringent. } \\
\text { Requirements such as emergency response } \\
\text { time or distance are more stringent. The } \\
\text { mandatory sites are given. }\end{array}$ \\
\hline Maximum coverage model & $\begin{array}{l}\text { Construction of the largest number of rescue sites due } \\
\text { to the control of rescue site construction costs to } \\
\text { maximize demand site coverage. }\end{array}$ & Limited construction cost. \\
\hline$P$-median model & $\begin{array}{c}\text { When the overall rescue level is limited, achieving } \\
\text { optimal average emergency rescue levels for all rescue } \\
\text { sites, such as shortest response time, shortest } \\
\text { distance, lowest cost, etc. }\end{array}$ & $\begin{array}{l}\text { Requirement of average emergency rescue } \\
\text { level is more stringent. }\end{array}$ \\
\hline$P$-center model & $\begin{array}{l}\text { Worst-case optimization of network emergency } \\
\text { rescue under a certain number of emergency rescue } \\
\text { stations, such as rescue the longest time to minimize, } \\
\text { rescue the longest distance to minimize, and } \\
\text { emergency maximum loss minimization. }\end{array}$ & $\begin{array}{l}\text { Adequate construction costs and high } \\
\text { requirements for rescue capacity. }\end{array}$ \\
\hline
\end{tabular}

considered, then the following problem can be solved by an integer programming model. Selecting $p$ - $t$ sites from alternative emergency rescue sites where each emergency rescue demand site is served by the nearest emergency rescue service site. The objective function use in such case will minimize the maximum service distance between all emergency demand sites and the sites that serve them The objective function:

$$
Z=\min \left\{\max _{u_{i} \in U}\left\{\min _{v_{j} \in X} d\left(u_{i}, v_{j}\right)\right\}\right\} .
$$

Constraints: s.t.

$$
\begin{aligned}
& d_{i j} x_{i j} \leq Z, \forall u_{i}, v_{j}, \\
& \sum_{v_{j \in V}} x_{i j} \geq 1, \forall u_{i}, \\
& \sum_{v_{j \in T}} y_{j}=t, \\
& \sum_{v_{j \in T}} y_{j}+\sum_{v_{j \notin T}} y_{j}=p, \\
& x_{i j}-y_{j} \leq 0, \forall u_{i}, v_{j}, \\
& x_{i j}, y_{j} \in\{0,1\}, \forall u_{i}, v_{j} .
\end{aligned}
$$

The objective function (4) represents the minimization of the maximum value of all minimum path lengths achieved from the rescue service site to the service demand site in the network.

Constraint (5) in which $Z$ denotes the maximum value of the rescue path length from the emergency rescue site to the demand site it covers.
Constraint (6) represents that the emergency rescue site in the network covers all emergency demand sites (at least one rescue site is covered).

Constraint (7) represents the set $T$ of mandatory emergency rescue sites, which are selected based on the characteristic indicators of complex network topology (such as degree value and betweenness centrality), and the number of mandatory emergency rescue sites is $t$.

Constraint (8) represents that the total number of emergency rescue sites is $p$, the number of mandatory emergency rescue sites is $t, p$ - $t$ emergency rescue service sites should be selected from emergency rescue alternative sites.

Constraint (9) represents that only the sites selected from the alternative sites or they are mandatory emergency rescue sites can carry out emergency rescue work.

Constraint (10) represents that $x_{i j}$ and $y_{j}$ is either 0 or 1 . When $x_{i j}$ takes 0 , it means that the site corresponding to $y_{j}$ is not set up as an emergency rescue service sites or is set up as an emergency rescue service sites but the rescue service does not covers $u_{i}$. When $x_{i j}$ takes 1 , it means that the site corresponding to $y_{j}$ is set up as an emergency rescue station and the rescue service cover $u_{i}$. When $y_{j}$ takes 0 , the site corresponding to $v_{j}$ is not set up as an emergency rescue service site. When $y_{j}$ takes 1 , the site corresponding to $v_{j}$ is set up as an emergency rescue service site.

3.3. Model Solving. The shortest distance between urban rail stations can be solved using Floyd algorithm. The algorithm is implemented through MATLAB programming. The indicators such as degree value and betweenness centrality can be solved by the equation, which is implemented by the PAJEK software. The $P$-center problem of emergency rescue service siting belongs to NP problem, and the model can be solved by a heuristic algorithm-genetic algorithm. The heuristic algorithm-genetic algorithm can be implemented 


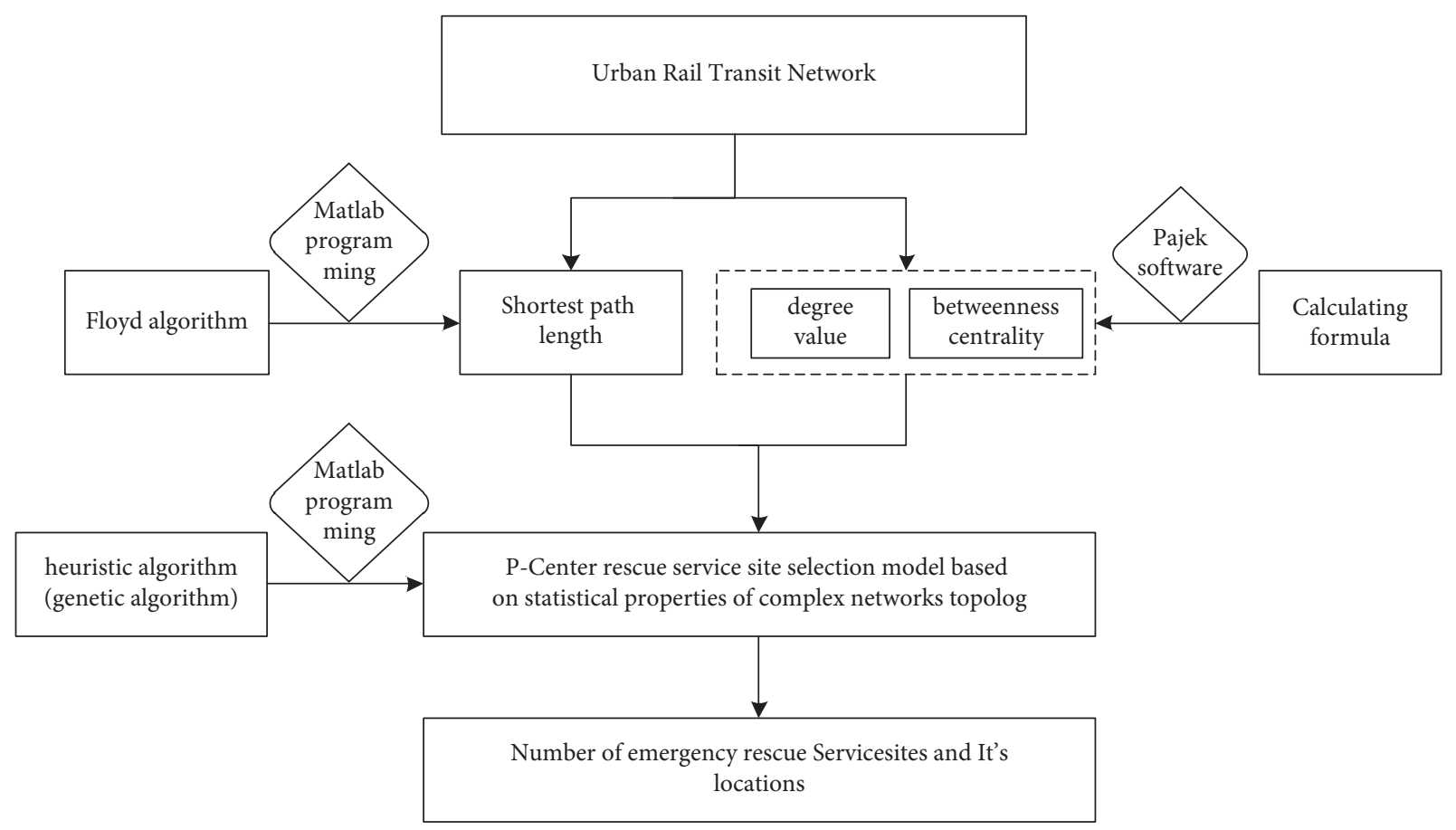

FIgURE 1: The solution process.

through MATLAB programming [19]. The solution process is shown in Figure 1.

\section{Example of Emergency Rescue Service Siting for Rail Transportation in Hangzhou}

The model is assessed by a real-world example rail transit of Hangzhou. Details are as follows.

4.1. Rail Network Topology Modeling. Until October 2021, there are nine operating lines of Hangzhou rail transit, including Line 1, Line 2, Line 4, Line 5, Line 6, Line 7, Line 8, Line 9, and Line 16. In order to present the integrity of the network, the aforementioned stations are that will be opened in the near future. Therefore, the rail transit, with all its lines, is considered appropriate for the evaluation. In the study, we treat the same interchange station of different lines as a network node, and select space $L$ method for topology abstraction of the network. A topology is constructed that forms an undirected and unprivileged rail network with 211 nodes. The topology diagram of Hangzhou rail transit network is shown in Figure 2.

4.2. Siting Nodes Selection. Node selection plays an important role in the assessment and analysis of optimization and scheduling models. The following steps were followed for the selection of siting nodes.

4.2.1. Shortest Path Length Calculation. The shortest path length between $211 * 211$ sites is solved by Floyd algorithm. The results show that the diameter of Hangzhou's rail network is 56, and the average path length between sites is
18.22 , and $50.5 \%$ of the sites can be reached within 17 stops. The sites contain a total of 17 stops. The shortest path length of the road network is shown in Figure 3.

4.2.2. Mandatory Sites Selection. The degree value and betweenness centrality of Hangzhou rail transit network are calculated by the Pajek software. The degree value, betweenness centrality, and their correlation were obtained by statistical analysis shown in Figure 4. Generally speaking, the degree value of the network nodes and the number of nodes show a positive correlation to a certain extent. However, this is not absolute; some nodes with smaller degree values have higher betweenness centrality. Therefore, the degree value, betweenness centrality, and the actual situation of the road network should be combined to determine the mandatory sites. For example, the betweenness centrality of Hangzhoudong Railway Station is 0.2995 , and the degree value is 5, the betweenness centrality of Pengbu Station is 0.2963 , and the degree value is 3 . In comparison, the Hangzhoudong Railway Station is more suitable as a mandatory rescue site. Although Pengbu Station ranks third in terms of betweenness centrality, because of the proximity of the Hangzhoudong Railway Station and Pengbu Station, Pengbu Station is not included in the collection of mandatory sites. After a comprehensive analysis, the mandatory emergency rescue sites for rail transportation in Hangzhou is computed (see Table 2).

4.2.3. Alternative Sites Selection. Hangzhou rail transit lines are set up with parking lots (vehicle sections) as shown in Table 3. According to the conditions of alternative site selection in section 3.2, it is determined that the 


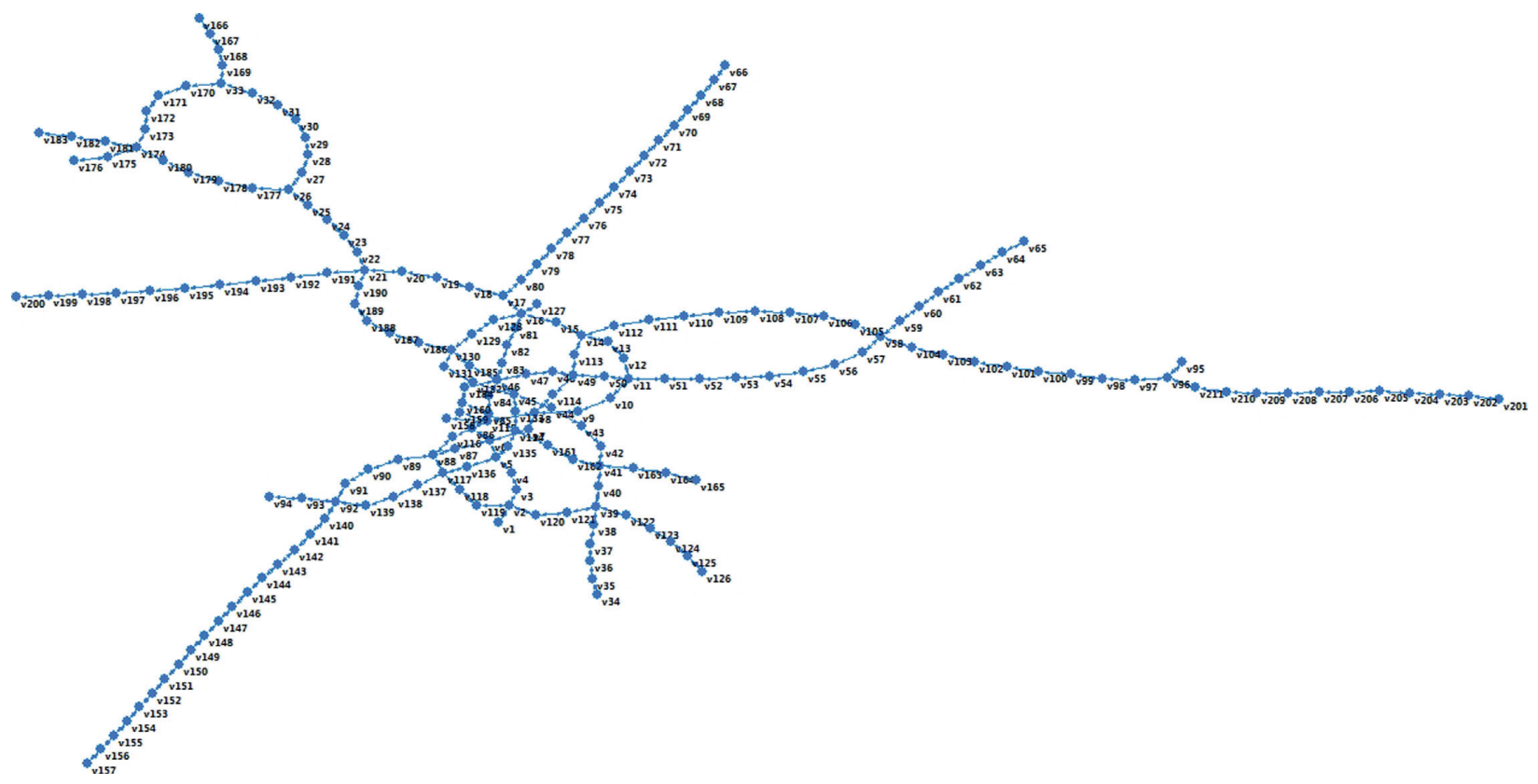

FIgURE 2: The topology diagram of Hangzhou rail transit network.

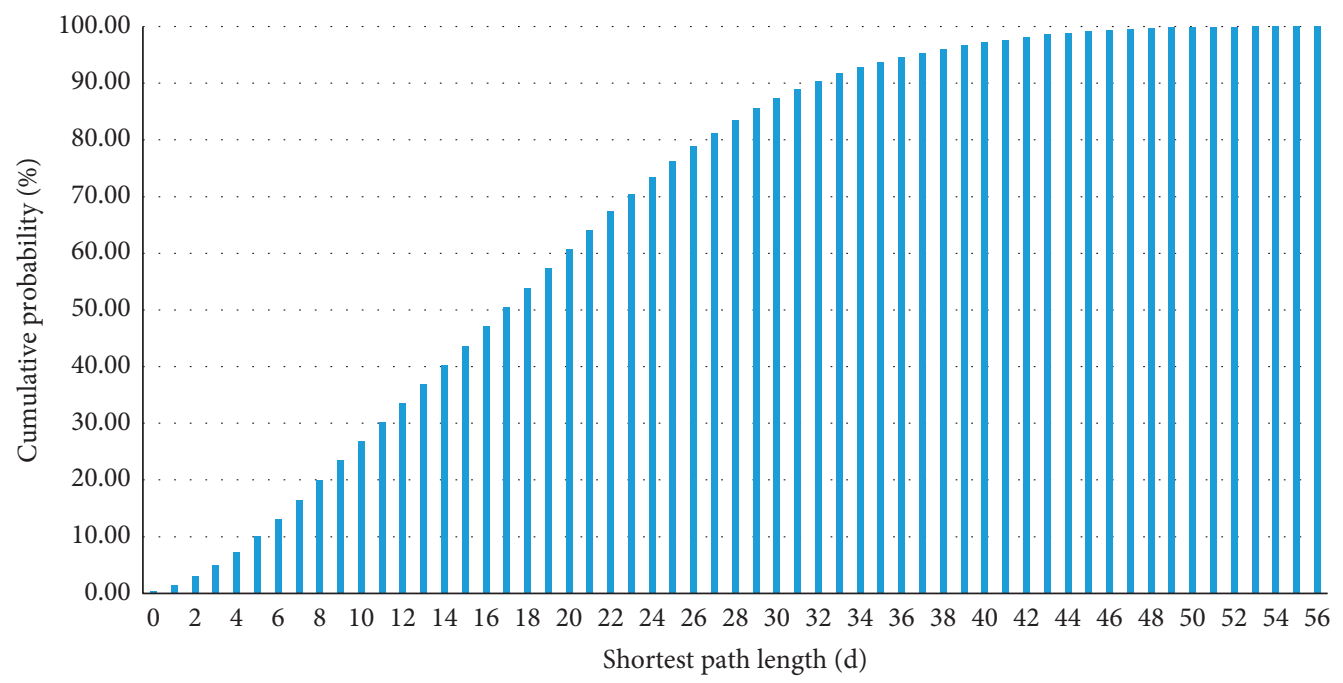

Figure 3: Distribution of the shortest path length of the topology for Hangzhou's rail network.

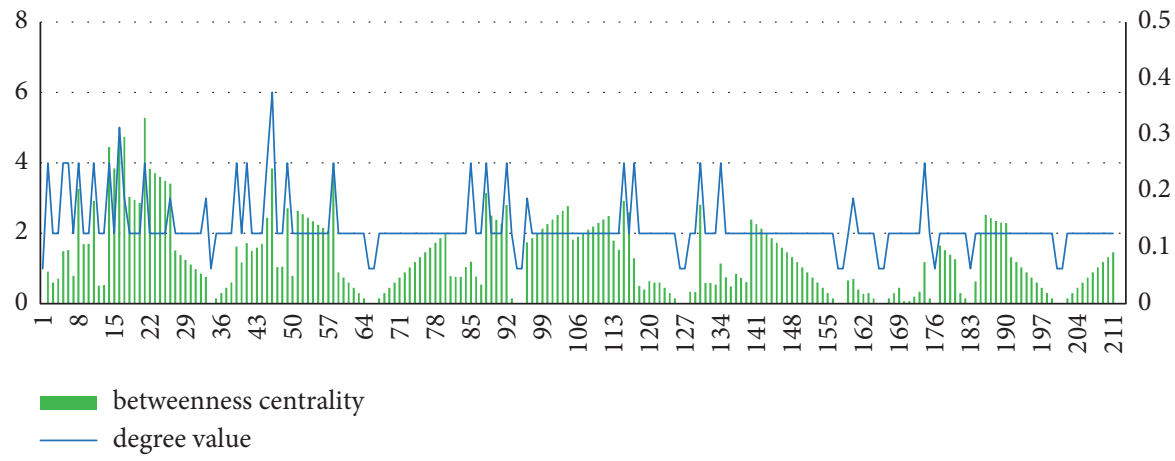

FIGURE 4: The correlation statistical analysis of degree value and betweenness centrality. 
TABLE 2: The mandatory emergency rescue sites.

\begin{tabular}{lccc}
\hline Station name & Line & Degree value & Betweenness centrality \\
\hline V46 (Qianjiang Road Station) & Line 2, Line 4, Line 8 & 6 & 0.2397 \\
V16 (Hangzhoudong Railway Station) & Line 1, Line 4, Line 6 & 5 & 0.2995 \\
V21 (Coach Center Station) & Line 1, Line 9 & 4 & 0.3297 \\
V58 (Sanba Station) & Line 2, Line 5 & 4 & 0.2436 \\
V8 (Chengzhan Station) & Line 1, Line 5 & 4 & 0.2035 \\
V85 (Citizen Center Station) & Line 4, Line 7 & 4 & 0.07478 \\
\hline
\end{tabular}

TABLE 3: Distribution of rail transit parking lots (vehicle sections) in Hangzhou.

\begin{tabular}{lcc}
\hline Line & Parking lots & Vehicle sections \\
\hline Line 1 & Qibao parking lot & Xianghu and Nanyang vehicle sections \\
Line 2 & Shuangqiao parking lot & Shushan vehicle section \\
Line 4 & Qibao parking lot & - \\
Line 5 & - & Wuchang and Guniangqiao vehicle sections \\
Line 6 & Fengbei parking lot & Shuangpu and Songjiatang vehicle sections \\
Line 7 & Jiangdongsan road parking lot & Yingzhong vehicle section \\
Line 8 & - & Xinwan vehicle section \\
Line 9 & Sibao parking lot & Changda road vehicle section \\
Line 10 & - & Renhe vehicle section \\
Line 16 & Jiangdongsan road parking lot & Shangquan vehicle section \\
\hline
\end{tabular}

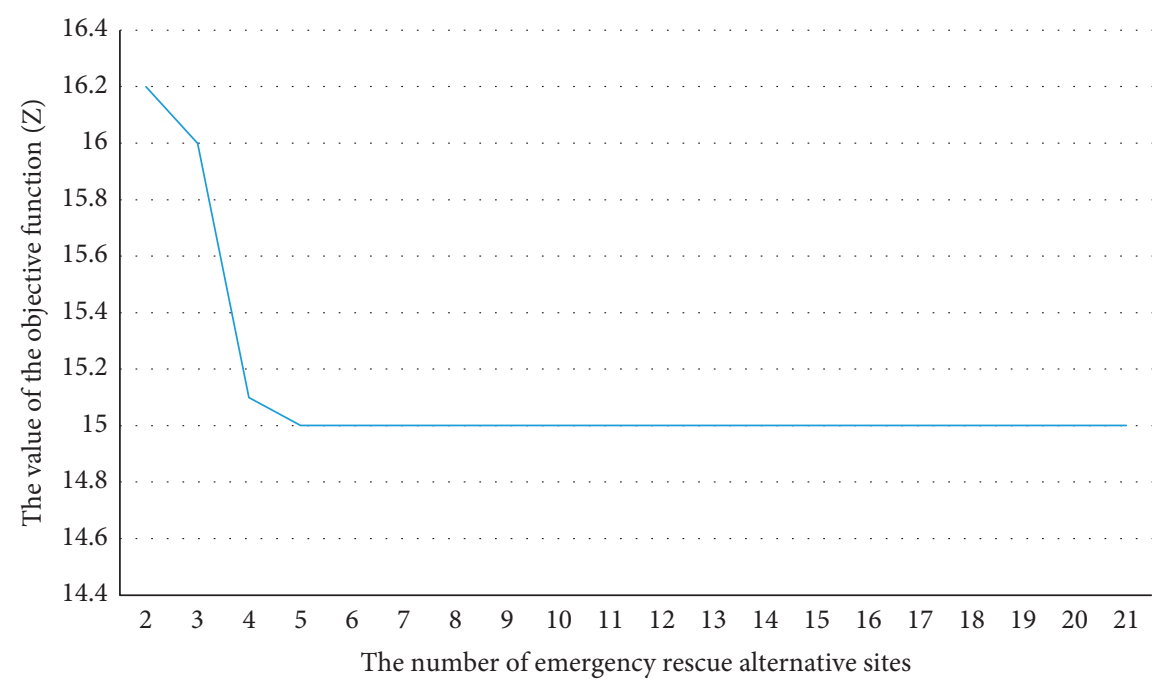

Figure 5: Modeling results.

alternative sites of Hangzhou rail transit emergency rescue are 21. The sites are, V1 (Xianghu Station), V11 (Fengqi Road Station), V17 (Pengbu Station), V18 (Qibao Station), V22 (Xiashaxi Station), V31 (Nanyang Station), V33 (Xiaoshan International Airport Station), V34 (Chaoyang Station), V96 (Lvting Road Station), V102 (Wuchang Station), V115 (Jiangcheng Road Station), V125 (Shuangqiao Station), V126 (Guniangqiao Station), V132 (Fengbei Station), V150 (Fuyang Coach Center Station), V157 (Shuangpu Station), V167 (Yingzhong Station), V176 (Jiangdong er Road Station), V183 (Xinwan Road Station), V200 (Longan Station), and V201 (Jiuzhou Street Station).
4.3. Optimal Siting Results. There are 211 emergency service demand sites, 21 emergency rescue alternative sites, and 6 emergency rescue mandatory sites. The model is solved by using genetic algorithm to achieve the optimal value of the objective function. The results obtained from the operations performed in MATLAB are graphically shown in Figure 5.

From the results of the operation, it can be seen that in the case of known set of mandatory and alternative rescue sites, when five sites are selected as emergency rescue service sites in the set of emergency rescue alternative sites, then the rescue distance maximum reaches minimum. The emergency rescue service sites setting that the Hangzhou rail transit can attain are V8 (Chengzhan Station), V16 
(Hangzhoudong Railway Station), V21 (Coach Center Station), V31 (Nanyang Station), V58 (Sanba Station), V46 (Qianjiang Road Station), V85 (Citizen Center Station), V96 (Lvting Road Station), V150 (Fuyang Coach Center Station), V176 (Jiangdong er Road Station), and V201 (Jiuzhou Street Station).

\section{Conclusion}

Urban rail transit system has become an essential mean of transportation and dispatching. Metropolitans from all over the world are focusing on the expansion and effective layout of transit systems to avail economic development. However, proper consideration need to be paid for the risk control measures and emergency rescue services in rail transit system to avoid the possibility of hazards, damages, and losses. With this research work, a model for the siting of urban rail transit emergency rescue service stations based on complex network characteristics is proposed. In the proposed method, the local and global statistical characteristic parameters of the complex network are selected for analysis. Besides, the shortest path lengths between nodes are selected as evaluation indexes in the sites selection of emergency rescue service. The genetic algorithm is used to solve the $P$ center siting model based on complex network characteristics. The algorithm minimizes the number of rescue sites while satisfying the optimal objective function and reduces the construction cost of rescue sites. The proposed model and the algorithms are verified by taking emergency rescue service siting in Hangzhou as a real-world example. The verification results show that the optimization model can provide a scientific basis for the decision related to the emergency rescue service siting. Moreover, the model has a significant effect on improving system reliability and reducing the risks of emergencies. As the network operation depends on equipment quality and maintenance system, an intelligent model is needed to evaluate the maintenance management of rail transit network. Our future strategy is to upgrade the model for the evaluation for the rail transit maintenance so that to ensure hazard-free operations of the network.

\section{Data Availability}

The data used to support the findings of this study are included within the article.

\section{Conflicts of Interest}

The authors declare that they have no conflicts of interest.

\section{References}

[1] M. S. Daskin and S. Owen, "Siting models in transportation," in Handbook of Transportation Science, R. W. Hall, Ed., , Kluwer Academic Publishers, Dordrecht, Netherlands, second edition, 2003.

[2] D. A. Valinsky, "Determination of the optimum siting of firefighting units in New York city," Journal of the Operations Research Society of America, vol. 3, no. 4, pp. 494-512, 1955.
[3] S. L. Hakimi, "Optimum sitings of switching centers and the absolute centers and medians of a graph," Operations Research, vol. 12, pp. 450-459, 1964.

[4] S. L. Hakimi, "Optimum distribution of switching centers in a communication network and some related graph theoretic problems," Operations Research, vol. 13, pp. 462-475, 1965.

[5] C. Toregas, R. Swain, and C. Revelle, "The siting of emergency service facilities," Operations Research, vol. 19, no. 16, pp. 1362-1373, 1971.

[6] Y. Liu, Y. Yuan, J. Shen, and W. Gao, "Emergency response facility location in transportation networks: a literature review," Journal of Traffic and Transportation Engineering, vol. 8, no. 2, pp. 153-169, 2021.

[7] X. Duan and L. I. Xiaowan, "Subway emergency resource location based on multi-objective estimation of distribution algorithm," Journal of Safety and Environment, vol. 19, no. 3, pp. 923-930, 2019.

[8] H. Wang and J. Zhou, "Location planning of railway rescue centers based on uncertain demand," Journal of Beijing Jiaotong University, vol. 19, no. 3, pp. 100-107, 2020.

[9] H. Fan, J. Guo, G. N. A Song, and X. Jiang, "Study on site selection of inland ports considering node failure under undecided demands and construction cost," Journal of Guangxi University(Natural Science Edition), vol. 45, no. 3, pp. 538-549, 2020.

[10] D. Peeters and I. Thomas, "The effect of spatial structure onpmedian results," Transportation Science, vol. 29, no. 4, pp. 366-373, 1995.

[11] S. Chai, Q. Liang, and S. Zhong, "Design of urban rail transit network constrained by urban road network, trips and landuse characteristics," Sustainability, vol. 11, no. 21, Article ID 6128, 2019.

[12] A. Fielbaum, S. J. Diaz, and A. Gschwender, "Optimal public transport networks for a general urban structure," Transportation Research Part B: Methodological, vol. 94, pp. 298313, 2016.

[13] S. P. Liu and J. Y. Yuan, "Emergency evacuation simulation method for urban rail transit accidents," Western China Communications Science \& Technology, vol. 1, pp. 146-149, 2019.

[14] L. Cadarso, E. Codina, L. F. Escudero, and A. Marín, "Rapid transit network design: considering recovery robustness and risk aversion measures," Transportation research procedia, vol. 22, pp. 255-264, 2017.

[15] X. Wang, L. Xiang, and G. Chen, Complex Network Theory and its Applications, Tsinghua University Press, Beijing, China, 2006.

[16] M. E. J. Newman and D. J. Watts, "Scaling and percolation in the small-world network model," Physical Review, vol. 60, no. 6, pp. 7332-7342, 1999.

[17] A. M. Caunhye, X. Nie, and S. Pokharel, "Optimization models in emergency logistics: a literature review," SocioEconomic Planning Sciences, vol. 46, no. 1, pp. 4-13, 2012.

[18] V. Blanger, A. Ruiz, and P. Soriano, "Recent optimization models and trends in Siting,Resiting and dispatching of emergency medical vehicles," European Journal of Operational Research, vol. 272, no. 1, pp. 1-23, 2019.

[19] C. Shan, A. Ning, D. Peng, and H. Zhang, "Backtracking algorithm with reduction for P-Center location Problem," Applicaiton Research of Computers, vol. 38, no. 3, pp. 734-737 + 769, 2021. 